The first paper describes the design and baseline characteristics of patients in the BPH Registry and Patient Survey in the USA. This important study will provide information on management practices and outcomes of men with this condition, as well as examining the effects of demographics, socio-economics, comorbidities and medical therapies.

Another important study in the USA is the Boston Area Community Health Survey, and in this issue the authors estimate the future magnitude of urological symptoms in that country. They found that it might reach the current level of cardiovascular disease and suggested that this will have huge affects on health service policy.

\title{
The Benign Prostatic Hyperplasia Registry and Patient Survey: study design, methods and patient baseline characteristics
}

Claus G. Roehrborn, James G. Nuckolls*, John T. Weit and William Steers未 on behalf of the BPH Registry and Patient Survey Steering Committee

Department of Urology, The University of Texas South-western Medical Center, Dallas, Texas, ${ }^{*}$ Carilion Medical Group, Galax, Virginia, +Department of Urology, The University of Michigan, Ann Arbor, Michigan, and FDepartment of Urology, University of Virginia, Charlottesville, Virginia, USA

Accepted for publication 12 March 2007

\section{Study Type - Symptom prevalence study (prospective cohort) \\ Level of Evidence 16}

OBJECTIVE

To describe the design and baseline cohort characteristics of the Benign Prostatic Hyperplasia (BPH) Registry and Patient Survey, an ongoing, prospective, observational, disease registry documenting management practices and patient outcomes in men in the USA with lower urinary tract symptoms associated with BPH (LUTS/BPH) in actual clinical practice settings.

\section{PATIENTS AND METHODS}

Men with LUTS/BPH who were either untreated or treated with $\alpha_{1}$-adrenergic blockers (ABs), $5 \alpha$-reductase inhibitors (5ARIs), a combination of these medications, or anticholinergics, and who met selection criteria, were enrolled at sites throughout the USA. At each visit, standardized forms and validated questionnaires were completed to assess the physicians' management practices and patients' clinical characteristics, sexual function, and health-related quality of life.

RESULTS

At the close of recruitment (February 2005), 6909 men (mean age 66.0 years) were enrolled at 402 sites by urologists and primary-care physicians. Before enrolment, $49 \%$ of the men were managed with watchful waiting (WW), 21\% with uroselective $A B$ monotherapy, $11 \%$ with non-uroselective $A B$ monotherapy, $6 \%$ with $5 \mathrm{ARI}$ monotherapy,

$11 \%$ with $A B+5 A R I$, and $2 \%$ with anticholinergics. After enrolment, 42\% were on WW and $26 \%$ were on selective $A B$ monotherapy; changes in other management groups were minimal. Overall, $33 \%$ of the men had mild, 52\% had moderate and 15\% had severe LUTS. The most common comorbidities were hypertension (53\%), high cholesterol (45\%) and sexual dysfunction (36\%).

\section{CONCLUSION}

The BPH Registry and Patient Survey will provide information on physician management practices and outcomes of 
men with LUTS/BPH, while examining the effects of demographics, socio-economics, comorbidities, and medical therapies.

\section{KEYWORDS}

prostatic hyperplasia, lower urinary tract symptoms, registries, outcome assessment, physician's practice patterns

\section{INTRODUCTION}

$\mathrm{BPH}$ is a common cause of morbidity in ageing men; depending on the degree of bother, men with BPH might complain of one or more LUTS, including increased frequency of urination, nocturia, hesitancy, urgency, straining, intermittency, dribbling, incomplete bladder emptying, and a weak urinary stream. The prevalence of histological $\mathrm{BPH}$ is 50\% in men aged $51-60$ years and increases to $90 \%$ in those aged $81-90$ years [1]. BPH can be a progressive disease that might cause serious complications, including haematuria, recurrent UTIs, acute urinary retention and, rarely, renal insufficiency $[2,3]$. LUTS are also associated with sexual dysfunction, bother, worry and interference with daily activities. The results of populationbased epidemiological studies show that LUTS are an independent risk factor for both erectile dysfunction (ED) and ejaculatory dysfunction $[4,5]$. Furthermore, men with moderate-to-severe LUTS report significant decreases in health-related quality of life, with 4-6 times the bother and interference with daily activities and twice the worry of men with mild LUTS [6]. Increased bother/decreased quality of life is the main reason that men seek help for LUTS associated with BPH (LUTS/BPH) and is a primary consideration in the treatment recommendations of the AUA BPH guideline [7]. Despite increased knowledge of the natural history and risk factors associated with LUTS/BPH, information on physicianpractice patterns, the longitudinal relationship between LUTS, sexual function and quality of life, and patient outcomes is limited.

A disease registry provides a systematic and inclusive database of information on representative individuals with an identified category of disease from a defined population [8]. A disease registry offers various differences in design and capability from a clinical trial. Unlike a clinical trial, which evaluates efficacy, a disease registry evaluates the effectiveness of management practices. Although a clinical trial provides data that can be useful for evidence-based recommendations on patient care, a disease registry provides data on patient care in actual practice settings, and changes in management over time. Whereas a clinical trial is conducted in a controlled setting, treatment is pre-specified by protocol, patients are evaluated at specified intervals, and enrolment is restricted by pre-specified inclusion and exclusion criteria, a disease registry documents actual care, treatments are not specified by protocol, patient visits are not controlled, and enrolment criteria are usually broad. A disease registry also offers an opportunity to assess whether physicians adhere to the practice guidelines recommended by speciality groups, societies and governmental organizations. Accordingly, data from a disease registry complement data from randomized clinical trials by providing clinicians, researchers, and policy makers with information on the actual implementation and effect of BPH treatments in a broader population of patients.

The BPH Registry and Patient Survey (BRPS), an ongoing, prospective, observational multicentre, disease registry, is collecting clinical and outcome data on men with LUTS/BPH who are managed by a geographically diverse group of urologists and primary-care physicians in the USA. This report describes the design and methods of the BRPS and baseline demographic and clinical characteristics of enrolled men and their LUTS/BPH management.

\section{PATIENTS AND METHODS}

The BRPS Steering Committee (see Acknowledgements), which comprises urologists, primary-care physicians, health services researchers, psychologists, and a biostatistician, was created to conceptualize the design and to oversee all scientific decisions on the conduct of the BRPS. A clinical research organization (i3 Research, Basking Ridge, NJ, USA) was contracted for data collection, database management and reporting. The BRPS is being conducted in accordance with Good Clinical Practice guidelines. Each principal investigator/ physician was required to submit registry documents and obtain Institutional Review Board approval before performing any registry-related procedures. The BRPS is funded by Sanofi-Aventis.

The overall objectives of the BRPS are: (i) to examine physician management practices and patient outcomes, including symptom amelioration and disease progression; (ii) to gain a better understanding of the relationship between LUTS and sexual dysfunction in ageing men; (iii) to further validate the Men's Sexual Health Questionnaire (MSHO) [9]; and (iv) to provide a benchmark for clinical outcomes against regional and national results. The registry was planned to include $\approx 500$ sites and $\approx 7500$ men. Based on long-term data on overall clinical disease progression and the failure of medical therapy from the Medical Therapy of Prostate Symptoms trial [10], participants are scheduled to be followed for $\geq 2$ years.

Physician management practices and patient characteristics and outcomes in the registry will be examined using descriptive statistics and various statistical models that address the relationship between a response/outcome and various exploratory variables. Clinical and quality-of-life measures will be assessed at baseline and at the follow-up, and expressed as the mean change from baseline. Analysis of covariance and regression models will be developed and the statistical significance of prognostic variables will be determined at the 0.05 level. Correlations between various clinical variables will be determined using Pearson product-moment and Spearman rank-order correlation. Demographic data and safety data will be summarized using descriptive statistics.

A geographically diverse group of USA urologists and primary-care physicians enrolled eligible men with LUTS/BPH (Fig. 1). We sought a ratio of urologists to primarycare physicians of $\approx 2: 1$; a maximum of 50 men could be enrolled at each investigational site. The primary inclusion criterion was a diagnosis of LUTS/BPH that was untreated (newly diagnosed or managed with watchful waiting, WW, or botanical products) or presently/recently treated with an $\alpha_{1}$ adrenergic blocker $(A B)$, a $5 \alpha$-reductase inhibitor (5ARI), a combination of an $A B$ and a $5 A R I$, or anticholinergics. 'Recently treated' was defined as ABs not discontinued $\geq 1$ month before enrolment and 5ARIs not discontinued $\geq 3$ months before enrolment. Patients were excluded if they had concomitant LUT disease or carcinoma, a 
FIG. 1. Investigational sites according to USA geographical region.

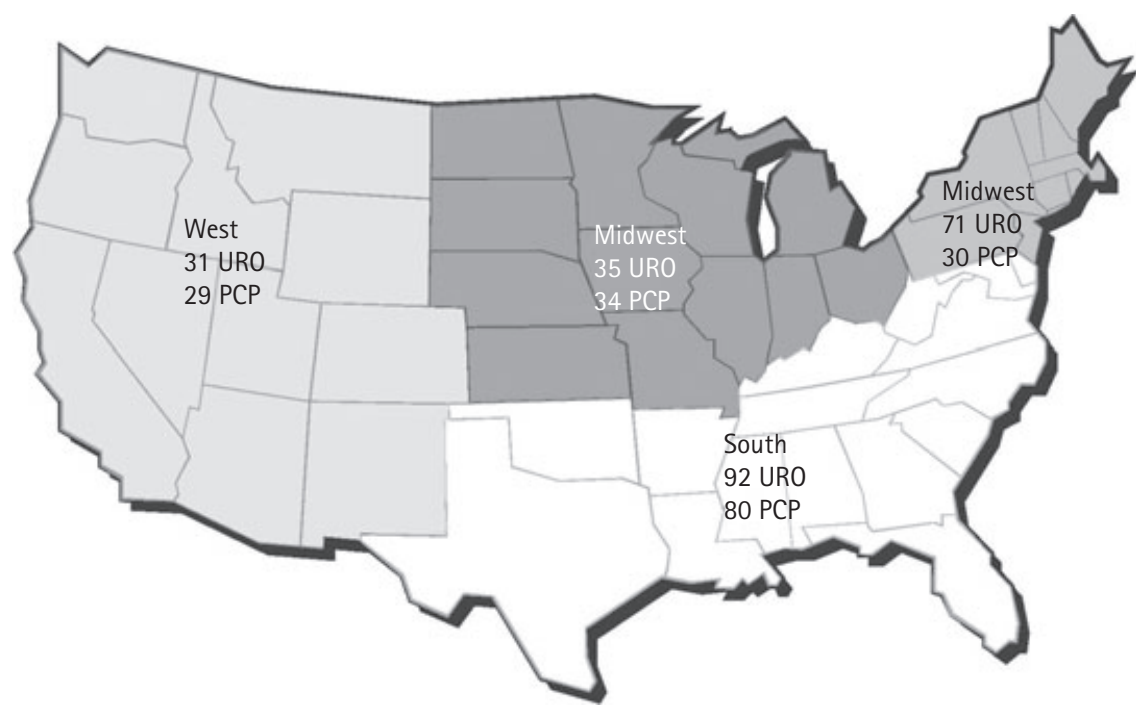

TABLE 1 The assessments at the initial and follow-up visits

\begin{tabular}{|c|c|c|c|c|}
\hline \multirow[b]{2}{*}{ Form/Questionnaire } & \multicolumn{3}{|l|}{ Visit 1} & \multirow[b]{2}{*}{ Follow-up visits } \\
\hline & Before enrolment & Baseline & After enrolment & \\
\hline \multicolumn{5}{|l|}{ Physician-completed } \\
\hline Physical Examination and & & $x$ & & $x$ \\
\hline \multicolumn{5}{|l|}{ Treatment Form } \\
\hline Clinical History Form & $x$ & & $x$ & $x$ \\
\hline Serious Adverse Event Form & & & & $x$ \\
\hline \multicolumn{5}{|l|}{ Patient-completed } \\
\hline Baseline Form & & $x$ & & \\
\hline Follow-up Form & & & & $x$ \\
\hline IPSS/IPSS Bother [11] & & $x$ & & $x$ \\
\hline BPH Impact Index [12] & & $x$ & & $x$ \\
\hline MSHO [9] & & $x$ & & $x$ \\
\hline SHIM [13] & & $x$ & & $x$ \\
\hline SF-12 [14] & & $x$ & & \\
\hline GAF & & $x$ & & $x$ \\
\hline
\end{tabular}

history of prostatic surgery, including minimally invasive procedures, isolated bladder neck disease or urethral stenosis/ strictures, gross haematuria, current acute urinary retention, neurological disease or other condition that has a direct effect on urinary function, acute renal disease, or active liver disease, were using LHRH analogues or antiandrogens, or were currently enrolled in a clinical or research study. Patients follow the management plan developed in collaboration with their physicians throughout the registry, with no treatments or patient clinic/office visits specified by protocol.
At enrolment (baseline visit), after explaining the protocol, obtaining informed consent, and providing the Health Insurance Portability and Accountability Act statement to enrolees, site investigators collected three types of data: (i) pre-enrolment data on current BPH management; (ii) baseline demographic and clinical information, overall assessments, and self-reported data on LUTS and LUTS bother, sexual function, and health-related quality of life using physician- and patient-completed forms and questionnaires; and (iii) data after enrolment on BPH management (Table 1) [9,11-14]. Follow-up visits are scheduled according to each physician's usual procedure. Once enrolled, patients could continue their pre-enrolment therapy or switch to another medical therapy or WW, according to decisions made by the physician and/or patient. We sought a sample in which about a third of men were managed with WW, about a third were presently/recently treated, and about a third were switched from WW to treatment.

At the baseline visit, each physician completed: (i) a Physical Examination and Treatment Form on each patient's LUTS/BPH history and any treatment prescribed or continued; and (ii) a Clinical History Form on comorbidities, concomitant medications, and any reported adverse events (unsolicited common complaints). Each patient completed: (i) a Baseline Form on sociodemographic information; (ii) selfadministered questionnaires, including the seven-item IPSS [11] (total score, 0-35, with scores of $\leq 7$ indicating mild, of 8-19 indicating moderate, and of $\geq 20$ indicating severe LUTS) and the IPSS Bother Question (score 0-6, with scores of $\leq 2$ indicating mild, of 3 indicating moderate, and of $\geq 4$ indicating severe bother); the four-item BPH Impact Index [12] (total score 0-12, with higher scores indicating a greater negative impact of BPH symptoms); the MSHO [9], which includes a three-item erection domain (total score 1-15, with lower scores indicating decreased function), an erection bother question (score range of $0-5$, with higher scores indicating greater bother), a sevenitem ejaculation domain (total score 1-35, with lower scores indicating decreased function), an ejaculation bother question (score 0-5, with higher scores indicating greater bother), and a six-item satisfaction domain (total score 6-30, with lower scores indicating decreased satisfaction); the fiveitem Sexual Health Inventory for Men [13] (SHIM or International Index of Erectile Function-5), with a total score of 5-25, with scores of $\leq 21$ indicating ED; the 12-Item Short Form Health Survey (SF-12) [14], with a Mental Component Score (MCS; score 0-100, with higher scores indicating better quality of life) and a Physical Component Score (PCS; score 0-100, with higher scores indicating better quality of life) that are standardized to a general USA population mean of $50 \pm 10$; and a Global Assessment Form (GAF) on the bother associated with urination problems and on satisfaction with sex life. 
At each follow-up visit, each physician completes: (i) a Physical Examination and Treatment Form on LUTS/BPH history and any treatment prescribed or continued or any surgery since the last visit; (ii) a Clinical History Form on comorbid conditions, concomitant medications, and any patientreported common complaints; and (iii) a Serious Adverse Event Form to describe any medical occurrence that results in death, is life-threatening, requires inpatient hospitalization or prolongation of existing hospitalization, results in significant disability/incapacity, is a congenital anomaly, or other medically important event. At each follow-up visit, each patient completes: (i) a Follow-up Form on health insurance, any discontinuations of medication, out-ofpocket expenses for treatment of urinary symptoms, and any visits to other doctors or the emergency room for urinary symptoms; and (ii) the self-administered IPSS, BPH Impact Index, MSHO, SHIM, and GAF. If a patient does not have an in-office follow-up visit within $6 \pm 1$ months of the last visit, the follow-up forms and questionnaires are mailed to the patient with instructions for their completion. A Final Status Form is completed by the physician when a patient completes (i.e. the investigator is satisfied that patient fulfils all registry requirements) or discontinues from the BPRS. In cases of discontinuation, the physician records the reason for discontinuation (e.g. did not meet inclusion/exclusion criteria, withdrawal of consent, adverse event, lost to follow-up, patient referred to another physician, new diagnosis of bladder or prostate cancer, patient enrolled in clinical or research study, investigator discontinued participation in registry), if known.

\section{RESULTS}

The first patient was enrolled on 23 January 2004 and the last on 25 February 2005. Enrolment in the BPRS was closed on 28 February 2005. At the time of the present baseline analysis (6 June 2005), data were available for 6909 men who were enrolled at 402 sites (Table 2), with 4537 (66\%) men seen by urologists and 2372 (34\%) seen by primary-care physicians. As of the present analysis, 634 (9\%) of the men had discontinued their participation in the registry, with withdrawal of consent (340 men) the most common reason for discontinuation.

\begin{tabular}{|c|c|c|}
\hline Variable (N) & $\begin{array}{l}N(\%) \text { patients or } \\
\text { baseline value }\end{array}$ & $\begin{array}{l}\text { TABLE } 2 \\
\text { Patient disposition }{ }^{*} \text { and the }\end{array}$ \\
\hline Patients enrolled & $6909(100)$ & demographic and socio- \\
\hline Patients discontinued & $634(9)$ & economic characteristics of \\
\hline \multicolumn{2}{|l|}{ Reason for discontinuation } & enrolled men at baseline \\
\hline Did not meet inclusion/exclusion criteria & $46(1)$ & \\
\hline Withdrawal of consent & $340(5)$ & \\
\hline Adverse event & $3(<1)$ & \\
\hline Death & $18(<1)$ & \\
\hline Lost to follow-up & $81(1)$ & \\
\hline Investigator discretion & $10(<1)$ & \\
\hline Referred to another physician & $2(<1)$ & \\
\hline New diagnosis prostate/bladder cancer & $50(1)$ & \\
\hline Enrolled in clinical/research study & $10(<1)$ & \\
\hline Investigator discontinued registry & $43(1)$ & \\
\hline Other & $31(<1)$ & \\
\hline Mean (SD) age, years (6787) & $66(10)$ & \\
\hline \multicolumn{3}{|l|}{ Age groups (6787) } \\
\hline$<50$ & $406(6)$ & \\
\hline $50-59$ & $1391(20)$ & \\
\hline $60-69$ & $2346(35)$ & \\
\hline $70-79$ & $2029(30)$ & \\
\hline$\geq 80$ & $615(9)$ & \\
\hline \multicolumn{3}{|l|}{ BMI, kg/m² (3236) } \\
\hline$<25$ & $733(23)$ & \\
\hline $25-29$ & $1460(45)$ & \\
\hline$\geq 30$ & $1043(32)$ & \\
\hline \multicolumn{3}{|l|}{ Race/ethnicity (6463) } \\
\hline White & $5379(83)$ & \\
\hline Black/African-American & $614(10)$ & \\
\hline Hispanic/White & $276(4)$ & \\
\hline Hispanic/Black & $35(1)$ & \\
\hline Other & $175(3)$ & \\
\hline \multicolumn{3}{|l|}{ Personal status, (6481) } \\
\hline Married/living with significant other & $5165(80)$ & \\
\hline Not married/living with significant other & $1316(20)$ & \\
\hline \multicolumn{3}{|l|}{ Educational status, (6355) } \\
\hline$<$ High school diploma & $578(9)$ & \\
\hline High school diploma/equivalent & $1669(26)$ & \\
\hline Some college & $1535(24)$ & \\
\hline$\geq$ College degree & $2573(40)$ & \\
\hline \multicolumn{3}{|l|}{ Annual household income, US\$ (6033) } \\
\hline$<15000$ & $537(9)$ & \\
\hline $15000-29999$ & $1033(17)$ & \\
\hline $30000-49999$ & $1416(23)$ & \\
\hline $50000-74999$ & $1330(22)$ & \\
\hline 75 000-149999 & $1335(22)$ & \\
\hline$\geq 150000$ & $382(6)$ & \\
\hline \multicolumn{3}{|l|}{ Primary health insurancet, (5062) } \\
\hline Private & $1613(32)$ & \\
\hline Medicare/supplemental & $3344(66)$ & \\
\hline Medicaid/veterans/military & $617(12)$ & \\
\hline Self-pay/none & $100(20$ & \\
\hline Drug coverage plan with co-pay & $592(12)$ & ${ }^{*}$ As of 6 June $2005 ;$ t Health \\
\hline Other & $327(6)$ & insurance categories are \\
\hline Do not know & $20(<1)$ & not mutually exclusive. \\
\hline
\end{tabular}




\begin{tabular}{|c|c|c|}
\hline Variable & No. patients & Baseline value \\
\hline \multicolumn{3}{|l|}{ Mean (SD): } \\
\hline time since first LUTS, years & 4595 & $5(5)$ \\
\hline time since BPH diagnosis, years & 5888 & $4(5)$ \\
\hline maximum urinary flow rate, $\mathrm{mL} / \mathrm{s}$ & 1045 & $12(7)$ \\
\hline Prostate size (DRE), $n(\%)$ & 5137 & \\
\hline normal & & $864(17)$ \\
\hline slightly enlarged & & $2248(44)$ \\
\hline moderately enlarged & & 1695 (33) \\
\hline significantly enlarged & & $330(6)$ \\
\hline total PSA, $\mathrm{ng} / \mathrm{mL}$ & 5420 & $2.9(4.4)$ \\
\hline total IPSS (range 0-35) & 6184 & $11.6(7.1)$ \\
\hline IPSS bother score (range 0-6) & 6415 & $2.5(1.5)$ \\
\hline BPH Impact Index (range 0-12) & 6450 & $2.8(2.8)$ \\
\hline Comorbidities, N (\%)* & 6284 & \\
\hline allergies/cold/flu/congestion & & $1070(15)$ \\
\hline arthritis & & $1357(20)$ \\
\hline depression/anxiety/sleep disorder & & $1117(16)$ \\
\hline diabetes & & $1196(17)$ \\
\hline digestive tract disorder & & $1427(21)$ \\
\hline general pain/inflammation & & 777 (11) \\
\hline heart disease/heart failure & & $1227(18)$ \\
\hline high cholesterol & & $3094(45)$ \\
\hline hypertension & & $3658(53)$ \\
\hline erectile or other sexual dysfunction & & $2511(36)$ \\
\hline
\end{tabular}

\begin{tabular}{|c|c|c|c|}
\hline Variable (range) & No. patients & Mean (SD) score & TABLE 4 \\
\hline \multicolumn{3}{|l|}{ MSHO [9] } & \multirow{7}{*}{$\begin{array}{l}\text { Sexual function and quality } \\
\text { of life of enrolled men at } \\
\text { baseline }\end{array}$} \\
\hline Erection domain (1-15) & 5786 & $8.4(4.4)$ & \\
\hline Erection bother (0-5) & 5785 & $3.6(1.3)$ & \\
\hline Ejaculation domain (1-35) & 5795 & $24.1(7.7)$ & \\
\hline Ejaculation bother (0-5) & 5534 & $4.0(1.2)$ & \\
\hline Satisfaction domain (6-30) & 5382 & $22.5(5.9)$ & \\
\hline SHIM [13] (5-25) & 5619 & $16.5(6.6)$ & \\
\hline SF-12 [14] & 3726 & & For all variables, a higher \\
\hline MCS (0-100) & & $52.2(9.2)$ & score indicates a better \\
\hline PCS $(0-100)$ & & $47.4(9.7)$ & outcome. \\
\hline
\end{tabular}

The mean (range) age of the enrolled men at baseline was 66.0 (30-94) years and most (83\%) were white (Table 2). Of 3236 men with body-mass index (BMI) data, 77\% had a BMI of $\geq 25 \mathrm{~kg} / \mathrm{m}^{2}$. Overall, $\approx 80 \%$ of the men were married or living with a partner, $64 \%$ had at least some education beyond a high school diploma, and $74 \%$ had household incomes of at least US\$30000.

At baseline, the mean time since the diagnosis of $\mathrm{BPH}$ was 4 (0-49) years, whereas the mean time since LUTS were first experienced was 5 (0-49) years; Table 3). Overall, 2065 (33\%) of the 6184 men who completed the IPSS had mild LUTS (IPSS $\leq 7), 3218$ (52\%) had moderate LUTS (8-19), and 901 (15\%) had severe LUTS $(\geq 20)$. Of 5137 men who had a DRE, 2025 (39\%) had a moderately or significantly enlarged prostate. Total PSA levels had a wide range of values (0.1$117.9 \mathrm{ng} / \mathrm{mL}$ ), with a median of $1.9 \mathrm{ng} / \mathrm{mL}$. In all, 6284 men (94\%) had at least one comorbid condition at the baseline visit.
The most common comorbidities were hypertension (53\%), high cholesterol (45\%) and sexual dysfunction (36\%) (Table 3).

Before enrolment, 6653 (96\%) of the 6909 men had data on their BPH management strategy, whereas 6667 (96\%) had data on their strategy after enrolment. Before enrolment, 3258 (49\%) of men with available data were managed with WW, 21\% with selective $A B s, 11 \%$ with non-selective $A B s$, $11 \%$ with $A B$ s and $5 A R I s, 6 \%$ with $5 A R I s$ and $2 \%$ with anticholinergics. At the end of the enrolment visit, $42 \%$ of the men were managed with $W W$, and the respective percentages were $26 \%, 11 \%, 12 \%, 7 \%$ and $2 \%$. Thus, the percentage of men who switched from WW to medical treatment at the baseline visit was only $7 \%$, which was less than the a priori estimate of 33\%. The percentage of men receiving a uroselective $A B$ (alfuzosin or tamsulosin) increased from 21\% before enrolment to 26\% after, whereas the percentage of men receiving each of the other $\mathrm{BPH}$ management options was essentially unchanged.

The mean SHIM score at baseline was 16.5 (Table 4), indicating that on average the men had mild-to-moderate ED. The mean MSHO erection domain score of 8.4 also suggested a mild-to-moderate degree of ED. Likewise, the mean $\mathrm{MSHO}$ bother score indicated that the men were 'a little bit' to 'moderately' bothered by their ED. ED was also reported as a common complaint by 1312 (50\%) of 2643 men with a complaint at the enrolment visit. The mean MSHO ejaculation domain score was 24.1, and the mean MSHO bother score was 4.0. Of 2643 men who reported a complaint at the baseline visit, 104 (4\%) reported abnormal ejaculation and 158 (6\%) reported reduced ejaculate volume. About half of men with a complaint of ED and twothirds of those with a complaint of ejaculatory dysfunction were receiving medical therapy for their LUTS/BPH.

The mean PCS and MCS of the SF-12 (Table 4) suggested that the men had a similar quality of life at baseline as a general USA population. Of 6318 men who completed the GAF before enrolment, 33\% reported that trouble with urination was 'not at all bothersome', 39\% reported that it 'bothers me a little', and 28\% indicated that it bothered them 'some' or 'a lot' during the past month. More men reported being satisfied with their sex lives than dissatisfied (43\% were 'very satisfied' or 
'mostly satisfied' with their sex lives, whereas $28 \%$ patients were 'mostly dissatisfied' or 'very dissatisfied').

\section{DISCUSSION}

At baseline, the men in the BPRS were predominantly aged $>50$ years, white, married and well-educated. Their common comorbidities include hypertension, high cholesterol and sexual dysfunction, with prevalence rates that are comparable to those in a population of USA men aged $\geq 50$ years $[4,15]$. Men enrolled in the registry had a broad range of LUTS severity (33\% mild, 52\% moderate and 15\% severe LUTS), which contrasts with most BPH clinical trials in which enrolment criteria pre-specify that men have moderate-to-severe LUTS. Thus, the men enrolled in the BPRS represent a USA population presenting with LUTS/BPH to urologists or primary-care physicians in everyday clinical practice, and can be longitudinally followed for their disease management and outcomes. Observation of this BPRS population might provide better insights on symptom progression, as a greater proportion of these men have mild LUTS than in standard BPH clinical trials. Only two other $\mathrm{BPH}$-related registries, which monitor results of specific procedures in a few patients, the European Association of Urology Real Life Data Registry with Transurethral Needle Ablation Therapy [16] and the international registry for an interstitial laser device [17], were reported previously.

As there are now more older men because of the ageing of the population and increased life-expectancy, appropriate management and use of resources for chronic diseases, including LUTS/BPH, have become major challenges for the healthcare system. This, together with a change from primarily surgical procedures to medical therapy as the first-line management approach for LUTS/BPH, and from predominantly urologists to primarycare physicians as the initial healthcare providers managing men with LUTS/BPH, has created a need for a better understanding of current physician practice patterns and patient outcomes.

The longitudinal BPRS was initiated to provide a comprehensive database of information on physician practice patterns and health outcomes of men with $\mathrm{BPH}$ who are initially managed conservatively with either WW or medical therapy. Although evidencebased treatment guidelines exist on the management of LUTS/BPH [7], data are needed on any differences between these recommendations and actual treatment decisions in clinical practice settings. The role of possible confounding variables, including demographics, socio-economics, comorbidities, and physician speciality, also needs to be explored. The database from the BPRS should complement data derived from clinical trials and provide a different and unique real-world perspective on management practices and outcomes for LUTS/BPH.

Limitations of the BPRS include a selection bias of physicians, that might have occurred because physicians enrolling men in the registry were not randomly selected but rather chose to participate. Selection bias is also a possibility for the men enrolled in the registry, but the prevalence rates of various comorbidities (e.g. hypertension, high cholesterol) in the enrolled men suggests that they are generally representative of an ageing USA male population $[4,15]$. Data from registries can also be limited by having too few patients receiving certain interventions or by patients with mild disease who do not warrant medical therapy.

In conclusion, the BPRS will provide a comprehensive database of information for assessing risk factors for LUTS/BPH, current physician-practice patterns in the management of LUTS/BPH, and health outcomes of men with LUTS/BPH in the USA.

\section{ACKNOWLEDGEMENTS}

The members of the BPH Registry \& Patient Survey Steering Committee are: Stanley E. Althof, Cleveland, Ohio; Steven Kaplan, New York, NY; Mark S. Litwin, Los Angeles, California; Michael Manyak, Washington, DC; Leonard S. Marks, Los Angeles, California; Kevin T. McVary, Chicago, Illinois; Charles Metzger, Glendora, California; Martin Miner, Swansea, Massachusetts; James Nuckolls, Roanoke, Virginia; Michael P. O'Leary, Boston, Massachusetts; David Pasta, San Francisco, California; Richard Payne, Encinitas, California; David Penson, Los Angeles,

California; Michael Perelman, New York, NY; Claus G. Roehrborn, Dallas, Texas; Raymond C. Rosen, Piscataway, New Jersey; Allen D. Seftel, Cleveland, Ohio; William Steers,
Charlottesville, Virginia; Alexis E. Te, New York, NY; John T. Wei, Ann Arbor, Michigan. The BPH Registry \& Patient Survey is supported by sanofi-aventis, and all members of the BPH Registry \& Patient Survey Steering Committee are paid consultants. Martin Miner, James Nuckolls, Richard Payne, William Steers, and John Wei also serve as site investigators for the BPH Registry \& Patient Survey. Patricia B. Leinen, PhD (Tri-Medical Communications, Media, PA) contributed to the preparation of the manuscript.

\section{CONFLICT OF INTEREST}

Claus G Roehrborn has received honoraria as Co-Chairman of the BPH Registry, and in the past was Investigator for Sanofi Aventis; John Wei is a Paid Consultant and a Study Investigator for Sanofi Aventis; William Steers is on the Advisory Board and is a Paid Consultant for Sanofi Aventis, a Study Investigator for Nouratis and Allergan, and a Paid Consultant for Dynogen.

\section{REFERENCES}

1 Berry SJ, Coffey DS, Walsh PC, Ewing LL. The development of human benign prostatic hyperplasia with age. $J$ Urol 1984; 132: 474-9

2 Emberton M, Andriole GL, de la Rosette $\mathrm{J}$ et al. Benign prostatic hyperplasia. a progressive disease of aging men. Urology 2003; 61: 267-73

3 Jepsen JV, Bruskewitz RC. Office evaluation of men with lower urinary tract symptoms. Urol Clin North Am 1998; 25: $545-54$

4 Rosen R, Altwein J, Boyle P et al. Lower urinary tract symptoms and male sexual dysfunction: the multinational survey of the aging male (MSAM-7). Eur Urol 2003; 44: 637-49

5 Blanker MH, Bohnen AM, Groeneveld FP et al. Correlates for erectile and ejaculatory dysfunction in older Dutch men: a community-based study. J Am Geriatr Soc 2001; 49: 436-42

6 Girman CJ, Epstein RS, Jacobsen SJ et al. Natural history of prostatism: impact of urinary symptoms on quality of life in 2115 randomly selected community men. Urology 1994; 44: 825-31

7 AUA Practice Guidelines Committee. AUA guideline on management of benign prostatic hyperplasia (2003). Chapter 1: diagnosis and treatment 
recommendations. J Urol 2003; 170: 530 47

8 Lubeck DP, Litwin MS, Henning JM et al. The CaPSURE database. a methodology for clinical practice and research in prostate cancer. CaPSURE Research Panel. Cancer of the Prostate Strategic Urologic Research Endeavor. Urology 1996; 48: 773-7

9 Rosen R, Catania J, Pollack L, Althof S, O'Leary M, Seftel AD. Male Sexual Health Questionnaire (MSHO): scale development and psychometric validation. Urology 2004; 64: 777-82

10 McConnell JD, Roehrborn CG, Bautista $\mathrm{OM}$ et al. The long-term effect of doxazosin, finasteride, and combination therapy on the clinical progression of benign prostatic hyperplasia. N Eng/ J Med 2003; 349: 2387-98

11 Barry MJ, Fowler FJ Jr, O'Leary MP et al. The American Urological Association symptom index for benign prostatic hyperplasia. The Measurement Committee of the American Urological Association. J Urol 1992; 148: 1549-57
12 Barry MJ, Fowler FJ Jr, O'Leary MP, Bruskewitz RC, Holtgrewe $\mathrm{HL}$, Mebust WK. Measuring diseasespecific health status in men with benign prostatic hyperplasia. Measurement Committee of the American Urological Association. Med Care 1995; 33: AS14555

13 Rosen RC, Cappelleri JC, Smith MD, Lipsky J, Pena BM. Development and evaluation of an abridged, 5-item version of the International Index of Erectile Function (IIEF-5) as a diagnostic tool for erectile dysfunction. Int J Impot Res 1999; 11: 319-26

14 Ware J Jr, Kosinski M, Keller SD. A 12-Item Short-Form Health Survey: construction of scales and preliminary tests of reliability and validity. Med Care 1996; 34: 220-33

15 National Center for Health Statistics. Prevalence of selected chronic conditions by age, sex and race. Trends in Health and Aging. Available at http://www.cdc.gov/ nchs/agingact.htm Accessed April 30 2007
16 Tubaro A. EAU Real Life Data Registry with TUNA therapy. Eur Urol Today 2004; 15: 5

17 Conn R, Muschter R, Adams C, Esch V. International registry results for an interstitial laser BPH treatment device. SPIE Proc 1996; 2671: 316-20

Correspondence: Claus G. Roehrborn, The University of Texas Southwestern Medical Center at Dallas, Department of Urology, 5323 Harry Hines Boulevard, J8-142, Dallas, TX 75390-9110, USA.

e-mail: claus.roehrborn@utsouthwestern. edu

Abbreviations: $A B, \alpha_{1}$-adrenergic blocker; 5ARI, 5 $\alpha$-reductase inhibitor; BMI, bodymass index; BRPS, BPH Registry and Patient Survey; GAF, Global Assessment Form; $E D$, erectile dysfunction; MSC, Mental Component Score; MSHO, Men's Sexual Health Questionnaire; PCS, Physical Component Score; SF-12, 12-Item Short Form Health Survey; SHIM, Sexual Health Inventory for Men; WW, watchful waiting. 